\title{
The novel use of oral antibiotic monotherapy in prosthetic valve endocarditis caused by Finegoldia magna: a case study
}

\author{
Siobhan Chien ${ }^{1 *}$ (1D, David Gorman ${ }^{1}$, Charilaos-Panagiotis Koutsogiannidis ${ }^{1}$, Ramanish Ravishankar ${ }^{2}$, \\ Ganesh Kamath ${ }^{3}$ and Vipin Zamvar ${ }^{1}$
}

\begin{abstract}
Background: Finegoldia magna, a Gram-positive anaerobic coccus, is part of the human normal microbiota as a commensal of mucocutaneous surfaces. However, it remains an uncommon pathogen in infective endocarditis, with only eight clinical cases previously reported in the literature.

Currently, infective endocarditis is routinely treated with prolonged intravenous antibiotic therapy. However, recent research has found that switching patients to oral antibiotics is non-inferior to prolonged parenteral antibiotic treatment, challenging the current guidelines for the treatment of infective endocarditis.

Case presentation: This case report focuses on a 52-year-old gentleman, who presented with initially culturenegative infective endocarditis following bioprosthetic aortic valve replacement. Blood cultures later grew Finegoldia magna. Following initial intravenous antibiotic therapy and re-do surgical replacement of the prosthetic aortic valve, the patient was successfully switched to oral antibiotic monotherapy, an unusual strategy in the treatment of infective endocarditis inspired by the recent publication of the POET trial. He made excellent progress on an eight-week course of oral antibiotics and was successfully discharged from surgical follow-up.

Conclusions: This case is the 9th reported case of Finegoldia magna infective endocarditis in the literature. Our case also raises the possibility of a more patient-friendly and cost-effective means of providing long-term antibiotic therapy in suitable patients with prosthetic valve endocarditis and suggests that the principles highlighted in the POET trial can also be applicable to post-operative patients after cardiac surgery.
\end{abstract}

Keywords: Finegoldia magna, Prosthetic valve endocarditis, Oral antibiotics, Administration, Cardiac surgical procedures, Treatment outcome, Infective endocarditis

\section{Background}

Finegoldia magna (previously known as Peptostreptococcus magnus) is the most commonly isolated Grampositive anaerobic cocci (GPAC) from clinical specimens, and is a normal commensal of human mucocutaneous surfaces $[1,2]$. However, it remains a rare and unusual microbe implicated in the pathogenesis of infective endocarditis.

\footnotetext{
* Correspondence: siobhan.chien@nhs.net

${ }^{1}$ Department of Cardiothoracic Surgery, Royal Infirmary of Edinburgh, 51

Little France Crescent, Edinburgh EH16 4SA, UK

Full list of author information is available at the end of the article
}

Although relatively rare, infective endocarditis carries a substantial morbidity and mortality risk, with current guidelines advocating the administration of prolonged, parenteral bactericidal therapy for complete infection eradication [3]. The recent publication of the Partial Oral Treatment of Endocarditis (POET) trial has recently proven switching patients with infective endocarditis to oral antibiotic therapy to be non-inferior to continued intravenous (IV) antibiotic therapy [4], challenging whether prolonged administration of parenteral antibiotics is necessary in a specific subset of patients with infective endocarditis.

(C) The Author(s). 2019 Open Access This article is distributed under the terms of the Creative Commons Attribution 4.0 International License (http://creativecommons.org/licenses/by/4.0/), which permits unrestricted use, distribution, and 


\section{Case report}

A 52-year-old man presented to our Emergency Department with sweats, lethargy and weakness 5 weeks following tissue aortic valve replacement with a $23 \mathrm{~mm}$ bioprosthetic valve. His background included severe aortic stenosis, mild left ventricular impairment, atrial fibrillation, hyperthyroidism, obstructive sleep apnoea and autistic spectrum disorder.

Following the original aortic valve replacement, the patient had made a good recovery, although he had been noted to be sweating at times. He maintained a normal white cell count and was apyrexial throughout, and was discharged home on postoperative day 6. Immediately following discharge, he did reattend via the Emergency Department with sweats and back pain, and was diagnosed with a lower respiratory tract infection.

Having re-presented with generalised weakness, lethargy and sweats, clinical examination was unremarkable and bloods demonstrated only a raised C-reactive protein (CRP) of 48. Trans-thoracic echocardiography demonstrated no evidence of endocarditis. Blood cultures taken on admission were initially reported as negative. However, during his admission the patient became pyrexial with worsening sweats and rigors. Urine culture, viral throat swab, CT scanning and dental review revealed no clear source of infection. There was no suggestion of immunocompromise or other risk factors for infection with this unusual pathogen.

Trans-oesophageal echocardiography on day 6 revealed infective endocarditis of the prosthetic aortic valve (see Fig. 1). Antibiotic cover was broadened to IV vancomycin, IV gentamicin and oral metronidazole. Pyrexia and inflammatory markers improved following this. IV gentamicin was later stopped on microbiology advice. Four days following admission, one set of blood cultures grew a Gram-positive anaerobe coccus, later identified as Finegoldia magna on day ten. Antibiotic sensitivities were assessed using gradient strip diffusion and are shown in Table 1.

Intravenous antibiotic therapy was commenced. The decision was taken to resort to surgical intervention with replacement of the infected prosthetic valve. The patient underwent re-do surgery with removel of the infected valve and implantation of another bioprosthetic valve. Culture and sensitivities from the excised valve were negative.

After 19 days of IV vancomycin and oral metronidazole, vancomycin was stopped. Given the excellent oral bioavailability of metronidazole, the decision was taken to treat the anaerobic infective endocarditis with oral metronidazole monotherapy. The patient's improving clinical condition, improving inflammatory markers and the achievement of source control through surgery supported the decision to switch to oral therapy.

The patient progressed well and was discharged on post-operative day 13 to complete a total 8-week course of oral metronidazole from the date of re-do surgery. Regular monitoring of CRP confirmed continuing improvement. He was discharged from follow-up eight weeks later, with final "test of cure" blood cultures reported as negative. No adverse effects following re-operation and prolonged antibiotic treatment were reported.

\section{Discussion}

Finegoldia magna, previously known as Peptostreptococcus magnus [5], is a Gram-positive anaerobic coccus (GPAC), and like other GPACs is a common commensal of human skin, mucous membranes, and gastrointestinal and genitourinary tracts [6-8].

Of the GPACs, F. magna is thought to be amongst the most pathogenic, and is a common isolate from skin and soft tissue infections, bone and joint infections, and diabetic foot infections $[7,9,10]$. Its particular virulence is

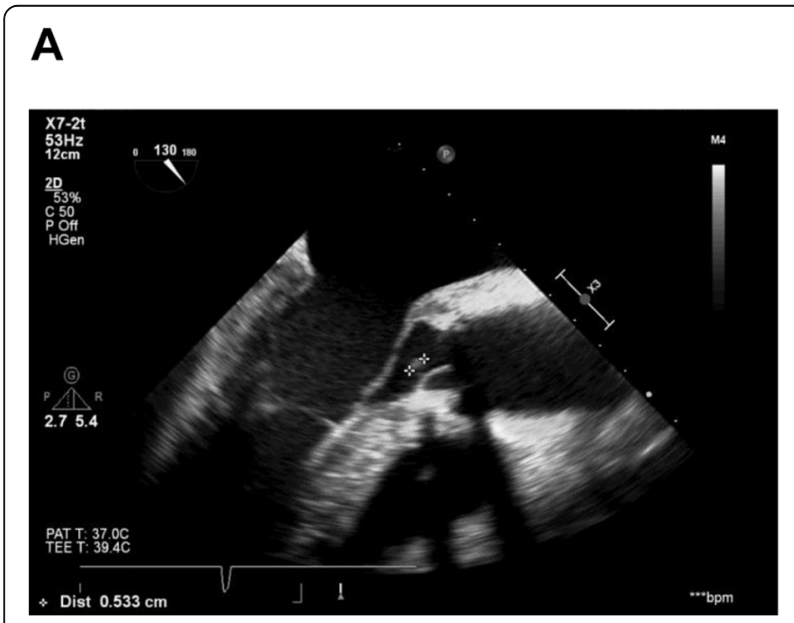

B

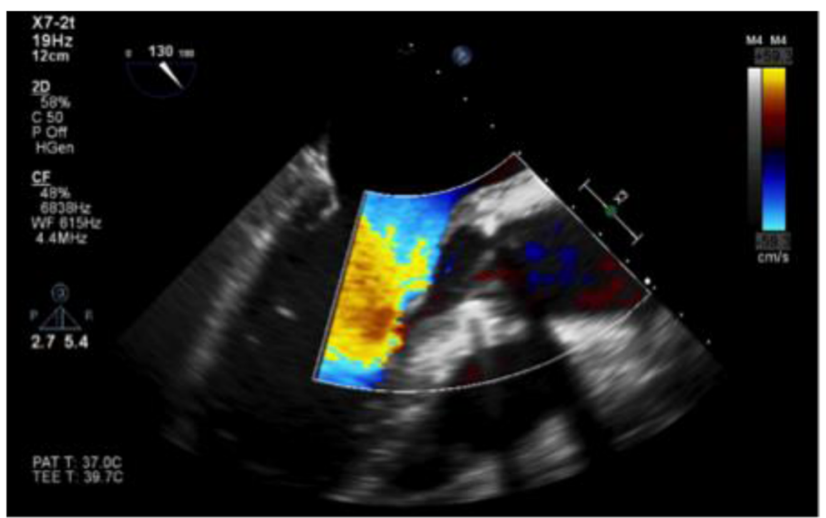

Fig. 1 Trans-oesophageal echocardiography (TOE) showing (a) location and size of vegetation and (b) mitral regurgitation 
Table 1 Antibiotic sensitivities of cultured F. magna. A lower MIC value indicates greater sensitivity to the tested antibiotic

\begin{tabular}{ll}
\hline Antibiotic tested & Minimum inhibitory concentration (MIC) \\
\hline Clindamycin & $0.064 \mathrm{mg} / \mathrm{L}$ \\
Co-amoxiclav & $0.500 \mathrm{mg} / \mathrm{L}$ \\
Metronidazole & $0.047 \mathrm{mg} / \mathrm{L}$ \\
Penicillin & $0.064 \mathrm{mg} / \mathrm{L}$ \\
Vancomycin & $0.094 \mathrm{mg} / \mathrm{L}$ \\
\hline
\end{tabular}

thought to be the product of a number of adaptations. Well described are: the expression of pili, which assist in adhesion and colonisation; the ability to bind to human albumin by way of a peptostreptococcal albumin-binding protein leading to accelerated growth rates; and expression of superantigens targeting B cells (the most common being Protein L) $[7,11,12]$.

Whilst the infections above due to F. magna appear to be relatively common, confirmed infective endocarditis due to $F$. magna is notably more rare. A search of the English-language literature reveals eight previously reported cases of endocarditis with $F$. magna or P. magnus as the confirmed causative organism.

Of note, blood cultures were negative in the majority of $F$. magna infective endocarditis cases, with definitive diagnosis made only by tissue specimens obtained during surgery [13]. Blood cultures yielded a positive microbiology result after four days in our case. Culturenegative infective endocarditis has a potentially negative impact on patient management, as this can lead to delays in the diagnosis and the initiation of the appropriate antibiotic regimen according to sensitivities [14]. Therefore, it is important to consider F. magna infection in a patient presenting with clinical suspicion of infective endocarditis after cardiac valvular surgery with initially sterile blood cultures [13].

According to guidelines from the European Society of Cardiology (ESC) and the American Heart Association (AHA), management of infective endocarditis classically involves the administration of intravenous antibiotics for up to 6 weeks' duration $[3,15]$. The majority of complications, including in-hospital mortality, are found to occur within the initial phase after admission [16-18]. A significant number of clinically stable patients are therefore subjected to a prolonged hospital admission after this initial phase to facilitate the administration of intravenous antibiotic therapy. Prolonged hospital stay is associated with an increased physical and psychological burden [19-22], whereas shorter inpatient admissions have better outcomes in other diseases and are more cost-effective [23-25]. The European and American guidelines consequently recommend the use of outpatient parenteral treatment in patients fulfilling certain criteria to alleviate the risks and complications associated with longer duration of hospital stay [3, 15, 26, 27].
However, the use of outpatient parenteral treatment is not without its challenges. For example, logistical issues must be addressed, patient and staff education is fundamental, close monitoring is required for efficacy and adverse effects, and patients must receive easy access to medical advice, in addition to adequate social and psychological support [4]. It has previously been hypothesised that the use of oral antibiotic therapy for the latter stages of the treatment period in stable patients with infective endocarditis would pose an appropriate alternative solution to these challenges, by allowing treatment to take place outside hospitals and without the need for intravenous catheters. However, this approach has been restricted due to the limited clinical evidence base for the safety and efficacy of oral antibiotic therapy for infective endocarditis [28-32].

The POET trial was published in the New England Journal of Medicine in 2018. 400 clinically stable patients with infective endocarditis on the left side of the heart were involved in this randomised unblinded trial, and were assigned to either continue intravenous treatment or to switch to oral antibiotic treatment, after the administration of at least 10 days of intravenous antibiotics. At the time of randomisation, at least 10 days of scheduled antibiotic treatment had to remain. The patients in the orally treated group were then discharged to outpatient treatment, if feasible. The POET trial confirmed that, in clinically stable patients with endocarditis on the left side of the heart, changing to oral antibiotic therapy was non-inferior to continued intravenous antibiotic therapy [4]. This supports the treatment of this patient with prolonged duration oral antibiotic therapy.

Previously, it had been suggested that intravenous antibiotic therapy was superior to the use of oral agents for infective endocarditis, with the main concern suggestive that gastric absorption of oral agents may be inadequate to allow sufficient plasma concentrations of antibiotics to facilitate bacterial killing [24]. The POET trial emphasises the importance of normal gastrointestinal function to facilitate the uptake of orally administered antibiotics [4]. The patient was commenced on IV vancomycin and oral metronidazole originally based on sensitivity results. Vancomycin must be administered via the intravenous route to adequately treat most infections, due to its poor oral bioavailability $(<10 \%)$ [33].

There is other evidence to support the choice of oral metronidazole monotherapy: oral metronidazole is known to have a high bioavailability (98.9\%) [34], with similar peak serum concentrations when administered via either the oral or intravenous route [35]. Oral metronidazole tablets are therefore often the preferred mode of administration, if tolerated. In this case, metronidazole was found to have a minimum inhibitory concentration of $0.047 \mathrm{mg} /$ $\mathrm{L}$, approximately $1 / 85$ th of the breakpoint concentration 
at which an organism is deemed metronidazole-sensitive, as per EUCAST guidelines current at the time of treatment [36]. This gave a good degree of confidence that metronidazole monotherapy would provide sufficient antimicrobial therapy.

Although clindamycin was indicated as another possible therapy by sensitivity testing, it is not usual practice in the UK to use clindamycin in the treatment of any bacterial endocarditis [37]. Furthermore, it was felt that a prolonged course of clindamycin was potentially harmful due to the significant associated risk of Clostridium difficile infection [38]. This supported the decision to treat with oral metronidazole alone.

In contrast to our case, the POET trial includes patients with endocarditis on the left side of the heart caused by Streptococcus, Enterococcus faecalis, Staphylococcus aureus, or coagulase-negative Staphylococci [4]. In the case of this patient, blood cultures were positive for F. magna, an anaerobic organism. Infective endocarditis caused by anaerobic pathogens remains relatively uncommon, and accounts for only $2-16 \%$ of all cases of infective endocarditis over the past four decades [39-42]. The POET trial did not include patients in whom an anaerobic pathogen was the causative organism. This case has demonstrated that switching to oral antibiotic therapy in a patient with an anaerobic infective endocarditis could also be satisfactory, but further research is required to prove if this statement could be substantiated with clinical evidence.

In addition, when switching to the oral route in the POET trial, all oral regimens included two antibiotics from different drug classes and with different antibacterial effects and different metabolization processes. This was to reduce the risk of pharmacokinetic variations of the orally administered antibiotics [43]. We have differed in offering only metronidazole as monotherapy, for the reasons outlined above and with a good degree of confidence that it would provide suitable cover. The POET trial has demonstrated that oral therapy with two agents was sufficient in the treatment of aerobic endocarditis in selected cases [4]. We have added to this evidence a case in which an anaerobic endocarditis was found to be treatable with oral monotherapy alone. In a time of renewed focus on antibiotic stewardship and avoidance of unnecessary use of antimicrobials, this is an exciting development.

Our patient received a total of 62 days of oral metronidazole, with only 6 days of IV metronidazole given during the immediate post-operative period in the intensive care setting, compared to only 19 days of IV vancomycin. Follow-up demonstrated clinical and biochemical evidence of cure. This suggests that the use of single agent oral antibiotic regimens may be a possible development in the future of infective endocarditis treatment. However, further research is required to determine the safety and efficacy of oral antibiotic monotherapy in the future.

\section{Conclusion}

We have discussed an unusual case of $F$. magna endocarditis affecting a bioprosthetic heart valve. Because of the tendency toward negative blood cultures in the early stages of diagnosis, a high index of suspicion is needed to diagnose endocarditis in vulnerable patients with non-specific features. This case also demonstrates successful treatment of an anaerobic endocarditis with outpatient oral antibiotic therapy. This builds on previous work which has shown outpatient oral antibiotics to be a feasible treatment for selected cases. Further research in this area may lead to practice changes that significantly reduce length of hospital stay, and its associated risks and costs, in these patients.

\section{Abbreviations \\ AHA: American Heart Association; AV: aortic valve; CRP: C-reactive protein; $C T$ : computed tomography; ESC: European Society of Cardiology; GPAC: Gram-positive anaerobic cocci; IV: intravenous; MIC: minimum inhibitory concentration; MV: mitral valve; POET trial: Partial Oral Treatment of Endocarditis trial}

\section{Acknowledgements}

We thank the patient for allowing us to present and analyse his clinical experience in this case report, and thank the staff within the Cardiothoracic Department at the Royal Infirmary of Edinburgh for their support in writing this case report. In particular, we wish to acknowledge Dr. Ewan Olson for sharing his expertise and knowledge in the area of cardiac microbiology.

\section{Authors' contributions}

$S$ Chien and D Gorman conceived of the presented idea, analysed the relevant literature and wrote the manuscript. CP Koutsogiannidis and V Zamvar provided critical feedback and supervised the findings. All authors read and approved the final manuscript.

\section{Funding}

Not applicable.

\section{Availability of data and materials}

Data sharing is not applicable to this article as no datasets were generated or analysed during the current study.

Ethics approval and consent to participate Not applicable.

\section{Consent for publication}

Written informed consent was obtained from the patient for publication of this case report.

\section{Competing interests}

The authors declare that they have no competing interests.

\section{Author details}

${ }^{1}$ Department of Cardiothoracic Surgery, Royal Infirmary of Edinburgh, 51 Little France Crescent, Edinburgh EH16 4SA, UK. ${ }^{2}$ University of Edinburgh, Edinburgh, UK. ${ }^{3}$ Department of Cardiothoracic Surgery, Kasturba Medical College, Manipal, India.

Received: 9 May 2019 Accepted: 10 September 2019

Published online: 18 September 2019

\section{References}

1. Murdoch DA. Gram-positive anaerobic cocci. Clin Microbiol Rev. 1998;11:81120.

2. Pouedras P, Donnio PY, Sire JM, et al. Prosthetic valve endocarditis and paravalvular abscess caused by Peptostreptococcus magnus. Clin Infect Dis. 1992;15:185. 
3. Baddour LM, Wilson WR, Bayer AS, et al. Infective endocarditis in adults: diagnosis, antimicrobial therapy, and Management of Complications. A scientific statement for professionals from the American Heart Association. Circulation. 2015:132(15):1435-86.

4. Iversen K, Ihlemann N, Gill SU, et al. Partial Oral versus intravenous antibiotic treatment of endocarditis. N Engl J Med. 2019;380:415-24.

5. Rosenthal ME, Rojtman AD, Frank E. Finegoldia magna (formerly Peptostreptococcus magnus): an overlooked etiology for toxic shock syndrome? Med Hypotheses. 2012;79(2):138-40.

6. Finegold SM. Anaerobic infections in humans: an overview. Anaerobe. 1995; 1(1):3-9.

7. Goto T, Yamashita A, Hirakawa $\mathrm{H}$, et al. Complete genome sequence of Finegoldia magna, an anaerobic opportunistic pathogen. DNA Res. 2008; 15(1):39-47

8. Basu P, Williams A, O'Brien MT, et al. A case of Finegoldia magna (formerly Peptostreptococcus magnus) infection mimicking disseminated malignancy. Int J Infect Dis. 2016:53:12-4.

9. Jneid J, Cassir N, Schuldiner S, et al. Exploring the microbiota of diabetic foot infections with culturomics. Front Cell Infect Microbiol. 2018:8:282.

10. Söderquist B, Björklund S, Hellmark B, et al. Finegoldia magna isolated from orthopaedic joint associated infections. J Clin Microbiol. 2017;55(11):3283-91.

11. Brüggemann $H$, Jensen $A$, Nazipi $S$, et al. Pan-genome analysis of the genus Finegoldia identifies two distinct clades, strain-specific heterogeneity, and putative virulence factors. Sci Rep. 2018;8:266.

12. Boyanova $L$, Markovska R, Miltov I. Virulence arsenal of the most pathogenic species among the gram-positive anaerobic cocci, Finegoldia magna. Anaerobe. 2016;42:145-51.

13. Hussein K, Savin Z, Shani L, et al. Infective endocarditis caused by Finegoldia magna following aortic dissection repair: a case report and data evaluation. Am J Case Rep. 2014;15:554-8.

14. Siciliano RF, Mansur AJ, Castelli JB, et al. Community-acquired culturenegative endocarditis: clinical characteristics and risk factors for mortality. Int J Infect Dis. 2014;25:191-5.

15. Habib G, Lancellotti P, Antunes MJ, et al. 2015 ESC guidelines for the management of infective endocarditis: the task force for the Management of Infective Endocarditis of the European Society of Cardiology (ESC). Eur Heart J. 2015;36(44):3075-128.

16. Dickerman SA, Abrutyn E, Barsic B, et al. The relationship between the iniaition of antimicrobial therapy and the incidence of stroke in infective endocarditis: an analysis from the ICE prospective cohort study (ICE-PCS). Am Heart J. 2007;154:1086-94.

17. Martín-Dávila P, Navas E, Fortún J, et al. Analysis of mortality and risk factors associated with native valve endocarditis in drug users: the importance of vegetation size. Am Heart J. 2005;150:1099-106.

18. Murdoch DR, Corey GR, Hoen B, et al. Clinical presentation, etiology, and outcome of infective endocarditis in the $21^{\text {st }}$ century: the international collaboration on endocarditis - prospective cohort study. Arch Intern Med. 2009;169:463-73.

19. Berg SK, Preisler P, Pedersen BD. Patients perspective on endocarditis - an intermezzo in life. Eur J Cardiovasc Nurs. 2010;9:126-31.

20. Verhagen DW, Hermanides J, Korevaar JC, et al. Health-related quality of life and posttraumatic stress disorder among survivors of left-sided native valve endocarditis. Clin Infect Dis. 2009:48:1559-65.

21. de Saint-Hubert M, Schoevaerdts D, Poulain G, et al. Risk factors predicting later functional decline in older hospitalised patients. Acta Clin Belg. 2009; 64:187-94.

22. Isaia G, Maero B, Gatti A, et al. Risk factors of functional decline during hospitalization in the oldest old. Aging Clin Exp Res. 2009;21:453-7.

23. Kehlet H. Fast-track colorectal surgery. Lancet. 2008;371:791-3.

24. Khoo CK, Vickery CJ, Forsyth N, et al. A prospective randomized controlled trial of multimodal perioperative management protocol in patients undergoing elective colorectal resection for cancer. Ann Surg. 2007;245: 867-72

25. Wind J, Polle SW, Fung Kon Kin PH, et al. Systematic review of enhanced recovery programmes in colonic surgery. Br J Surg. 2006;93:800-9.

26. Andrews MM, von Reyn CF. Patient selection criteria and management guidelines for outpatient parenteral antibiotic therapy for native valve infective endocarditis. Clin Infect Dis. 2001:33:203-9.

27. Lacroix A, Revest M, Patrat-Delon S, et al. Outpatient parenteral antimicrobial therapy for infective endocarditis: a cost-effective strategy. Med Mal Infect. 2014;44:327-30.
28. Al-Omari A, Cameron DW, Lee C, et al. Oral antibiotic therapy for the treatment of infective endocarditis: a systematic review. BMC Infect Dis. 2014; $14: 140$

29. Heldman AW, Hartert TV, Ray SC, et al. Oral antibiotic treatment of right-sided staphylococcal endocarditis in injection drug users: prospective randomized comparison with parenteral therapy. Am J Med. 1996;101:68-76.

30. Dworkin RJ, Lee BL, Sande MA, Chambers HF. Treatment of right-sided Staphylococcus aureus endocarditis in intravenous drug users with ciprofloxacin and rifampicin. Lancet. 1989;2:1071-3.

31. Iversen $\mathrm{K}, \mathrm{H} ø \mathrm{st} \mathrm{N}$, Bruun NE, et al. Partial oral treatment of endocarditis. Am Heart J. 2013:165:116-22.

32. Mzabi A, Kernéis S, Richaud C, Podglajen I, Fernandez-Gerlinger MP, Mainardi JL. Switch to oral antibiotics in the treatment of infective endocarditis is not associated with increased risk of mortality in nonseverely ill patients. Clin Microbiol Infect. 2016;22:607-12.

33. Butler-Laporte G. De L'Étoile-Morel S, Cheng MP, McDonald EG, Lee TC. MRSA colonization status as a predictor of clinical infection: a systematic review and meta-analysis. J Inf Secur. 2018;77(6):489-95.

34. Lee LL, Rowland J, Ling CHY, Fiakos E. A quantity survey of intravenous administration of metronidazole in its different forms in a tertiary teaching hospital. Intern Med J. 2010;40(8):592-6.

35. Ralph ED. Clinical pharmacokinetics of metronidazole. Clin Pharmacokinet. 1983;8(1):43-62

36. The European committee on antimicrobial susceptibility testing (EUCAST). Breakpoint tables for interpretation of MICs and zone diameters. Version 8.1. 2018. Available from: www.eucast.org.

37. Elliott TS, Foweraker J, Gould FK, Perry JD, Sandoe JA. Working Party of the British Society for antimicrobial chemotherapy. Guidelines for the antibiotic treatment of endocarditis in adults: report of the working Party of the British Society for antimicrobial chemotherapy. J Antimicrob Chemother. 2004;54(6):971-81.

38. Pan J, Kavanagh K, Marwick C, et al. Residual effect of community antimicrobial exposure on risk of hospital onset healthcare associated Clostridioides difficile infection: a case-control study using national linked data. J Hosp Infect 2019 Jun 4. doi: https://doi.org/10.1016/j.jhin.2019.05.016. [Epub ahead of print].

39. Feiner JM, Dowell VR Jr. Anaerobic bacterial endocarditis. N Engl J Med. 1970;283:1188-92

40. Sapico FL, Sarma RJ. Infective endocarditis due to anaerobic and microaerophilic bacteria. West J Med. 1982;137(1):18-23.

41. Von Reyn CF, Levy BS, Arbelt RD, et al. Infective endocarditis: an analysis based on strict case definitions. Ann Intern Med. 1981;94:505-18.

42. Wilson WR, Geraci JE. Anaerobic infections of the cardiovascular system. First United States metronidazole conference. Eds Finegold SM et al. Biomedical information information Corp. NY. 319-330. Proceedings from a symposium, Tarpon Springs, Florida, February 19-20, 1982

43. Bassetti S, Laifer G, Goy G, et al. Endocarditis caused by Finegoldia magna (formerly Peptostreptococcus magnus): diagnosis depends on the blood culture system used. Diagn Microbiol Infect Dis. 2003;47:359-60.

\section{Publisher's Note}

Springer Nature remains neutral with regard to jurisdictional claims in published maps and institutional affiliations.

Ready to submit your research? Choose BMC and benefit from

- fast, convenient online submission

- thorough peer review by experienced researchers in your field

- rapid publication on acceptance

- support for research data, including large and complex data types

- gold Open Access which fosters wider collaboration and increased citations

- maximum visibility for your research: over $100 \mathrm{M}$ website views per year

At BMC, research is always in progress.

Learn more biomedcentral.com/submissions 